\title{
Reviewing the literature: adopting a systematic approach
}

\author{
Julie Bruce, BSc, MSc, Research Fellow in Epidemiology; Jill Mollison, BSc, PhD, Senior Lecturer in Medical Statistics, \\ Department of Public Health, University of Aberdeen, Aberdeen, UK
}

Correspondence: : Ms Julie Bruce, Department of Public Health, Medical School, University of Aberdeen, Polwarth Building, Foresterhill, Aberdeen AB25 2ZD, UK. E-mail: j.bruce@abdn.ac.uk

\section{(Accepted29Qtdber2003)}

Journal of Family Planning and Reproductive Health Care 2004; 30(1): 13-16

\section{Introduction}

Review articles are often commissioned or submitted for publication in the Journal of Family Planning and Reproductive Health Care. Review papers summarise current knowledge and often attempt to reconcile conflicting scientific results from individual primary studies. As such, they are an essential resource for busy practitioners and researchers who struggle to keep up with the accumulating, ever-increasing body of evidence. However, many review manuscripts submitted to the Journal are based upon expert opinion rather than objective, systematic appraisal of the literature. The aim of this paper is to highlight the strength of a systematic review approach to reviewing the literature over traditional narrative reviews. In addition, this paper also makes the distinction between systematic reviews and meta-analysis. Regardless of the research question, practitioners should adopt a systematic approach to literature searching, critical appraisal and interpretation of data from primary studies. This paper outlines the crucial steps for consideration and acts as a guide for practitioners conducting reviews in family planning and reproductive health. These criteria may be considered specific to reviews of therapeutic interventions adopting a randomised controlled trial (RCT) design. However, this standard approach can be adopted and modified for reviews addressing different questions and/or study designs, e.g. evaluation of observational studies, diagnostic and screening tests ${ }^{1}$ or prognostic variables. ${ }^{2}$ Additionally, the criteria outlined in this paper should enable Journal readers to develop a critical approach to reading published systematic reviews.

\section{What is a systematic review?}

A systematic review is a critical synthesis of research evidence, which involves analysis of all available and relevant evidence in a systematic, objective and robust manner. A formal, rigorous methodological process is followed (Figure 1). This includes clarification of the research question, identification of eligible literature using electronic bibliographic databases and other information sources, assessment of study quality, data extraction, summarising and interpretation of results. A common misconception is that systematic reviews can only be conducted on RCTs. Systematic review methodology can be adapted to address a wide range of questions and the study design of the included studies will be dictated by the research question.

In the 1970s, Archie Cochrane highlighted the lack of critical summaries for use by health care professionals involved in decision making. ${ }^{3}$ This led to the development of the Cochrane Collaboration, an international network of health care professionals who prepare and update systematic reviews. Many other research groups and organisations conduct and produce systematic reviews [e.g. National Health Service Centre for Reviews and
Dissemination (NHS CRD), National Institute for Clinical Excellence (NICE)]. Systematic reviews use objective, explicit and transparent methods, which allow the reader to follow how conclusions were reached. They may indicate that further primary research is required in areas where no evidence exists or if current evidence is inconclusive. Findings from systematic reviews are used to inform health care policy decisions and practice-based recommendations based on available evidence.

Terminology: systematic review and meta-analysis A systematic review may or may not incorporate a metaanalysis, depending upon whether statistical 'pooling' of results is appropriate or feasible. Meta-analysis is a quantitative method for combining the results of several independent studies that measure the same outcome so that an overall summary statistic can be calculated. Therefore, the term systematic review is not synonymous with the term meta-analysis. Whereas a systematic review may provide an overview of whether an intervention works, a systematic review with meta-analysis would provide an estimate of the magnitude of effect for that particular

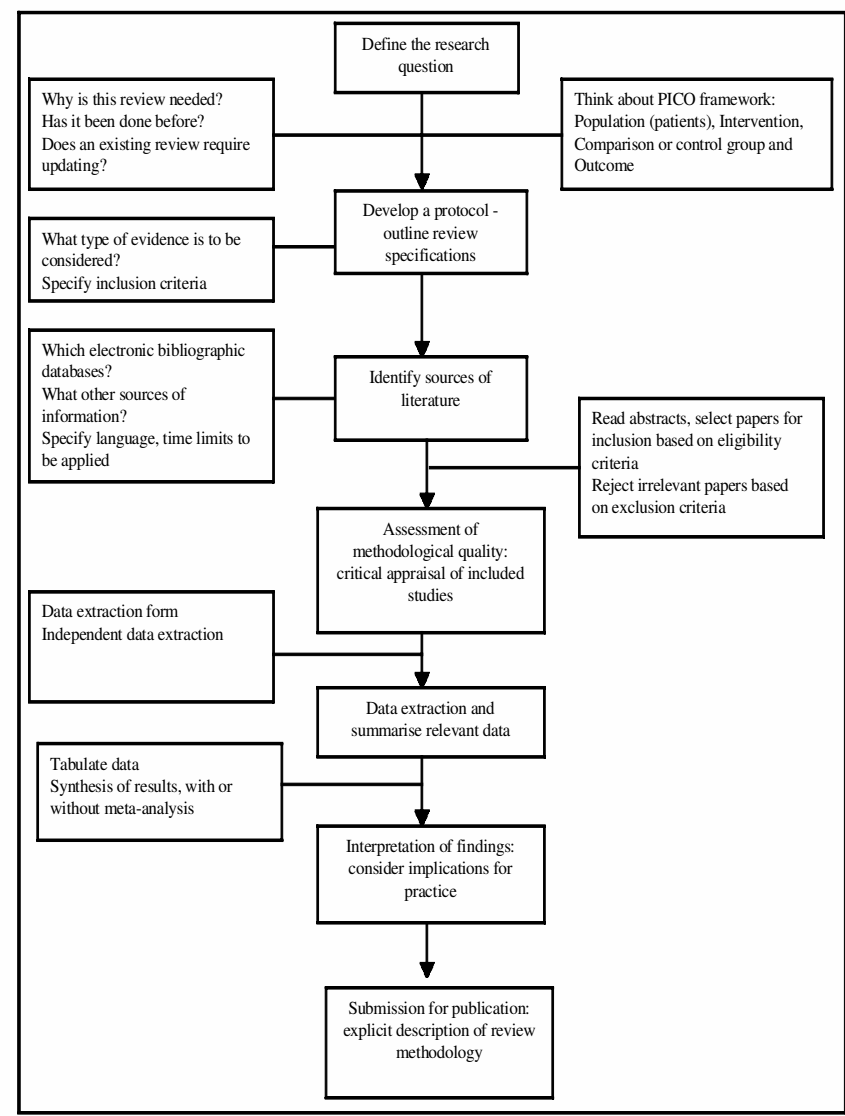

Figure 1 Flow chart of process of conducting a systematic literature review 
intervention. Another common misconception is that systematic reviews must include a meta-analysis. In fact, it may not always be appropriate, and may be misleading, to numerically combine studies in a meta-analysis. Furthermore, the application of a meta-analysis does not necessarily mean that a systematic review has been conducted.

\section{What is wrong with narrative reviews?}

Traditional narrative reviews can be an unreliable source of information and be subject to error and bias (systematic error). Narrative reviews often fail to identify, include or appraise all available evidence or specify the process by which judgements, conclusions and recommendations are made. Conclusions drawn from a given body of evidence may be more associated with the expertise or influenced by personal opinion of the reviewer than with the available data. ${ }^{4}$ It is possible to selectively find evidence to support your own argument and prejudices. For example, experts invited to write narrative reviews on the same topic often come to opposing conclusions.

\section{Conducting a systematic review}

\section{Defining your research question}

A crucial step in any review is to clearly define the question to be addressed. Does your question relate to assessment of treatment, diagnosis, risk or prognosis? Which particular patient groups or settings are you interested in? Which interventions and/or comparisons are of interest? Consideration of these simple steps when formulating your review question will help focus your aims and objectives. ${ }^{5}$ The 'PICO' (Population, Intervention, Control, Outcome) framework, widely used in evidence-based medicine, may help focus your research question, particularly when appraising therapeutic interventions. Crilly and Foy ${ }^{6}$ recently outlined the key components for an evidencebased approach to answering clinical questions.

Writing a short 'protocol' or summary plan of the process for your review can also keep you focused. Once you have considered the key components of your research question, think of appropriate sources of information to answer your question. Which electronic bibliographic databases are relevant for your topic? What types of studies are you interested in (e.g. RCTs, cohort or case-control studies)? Searching for controlled clinical trials in a systematic review of the prevalence of chlamydia would clearly be inappropriate. This is an epidemiological question and would require data from cross-sectional or cohort studies from different populations and settings. You should bear in mind hierarchies of evidence, whereby experimental studies are more rigorous and less subject to bias and confounding than observational study designs. Observational studies have been shown to produce strong, but biased, estimates of effect and the strength of association can vary across study design and methodological quality. ${ }^{7}$

\section{Literature searching}

A systematic literature review aims to consider all available and eligible evidence; therefore, finding all the evidence is a key component of conducting a comprehensive literature review. Consider which electronic bibliographic databases are most relevant to your clinical topic. The two major biomedical bibliographic databases are MEDLINE and EMBASE. In addition, the Cochrane Library comprises multiple databases, including registers of controlled clinical trials and databases of completed and ongoing systematic reviews. This is often a useful place to begin your search to identify whether a systematic review has been already conducted or is currently underway. Another
Table 1 Sample of electronic bibliographic databases specific to health care

\begin{tabular}{ll}
\hline Bibliographic database & Topics covered (website address) \\
\hline MEDLINE, EMBASE & $\begin{array}{l}\text { Medicine, nursing, dentistry, toxicology, } \\
\text { pharmacology and health care delivery }\end{array}$ \\
The Cochrane Library & $\begin{array}{l}\text { Incorporates multiple databases. Covers } \\
\text { evidence of effectiveness of health care } \\
\text { interventions }\end{array}$ \\
Science Citation Index (SCI) & Medicine, science and technology \\
Cumulative Index to Nursing & $\begin{array}{l}\text { Nursing, consumer health, complementary } \\
\text { medicine, professions allied to health }\end{array}$ \\
PschLIT Allied Health (CINAHL) & $\begin{array}{l}\text { Psychology, psychiatry and neuroscience } \\
\text { literature }\end{array}$ \\
CANCERLIT & $\begin{array}{l}\text { Produced by National Cancer Institute } \\
\text { (http://www.cancer.gov/cancer_- }\end{array}$ \\
information/cancer_literature/) \\
Health Economics \\
Evaluation Database (HEED) \\
$\begin{array}{l}\text { Health economics literature } \\
\text { (http://www.ohe-heed.com/) } \\
\text { Medicine (AMED) }\end{array}$ & $\begin{array}{l}\text { Also covers occupational therapy, } \\
\text { physiotherapy, palliative care and } \\
\text { rehabilitation }\end{array}$ \\
Population information & $\begin{array}{l}\text { Reproductive health, family planning } \\
\text { developing country literature, (http://db. } \\
\text { jhuccp.org/popinform/basic.html) }\end{array}$ \\
\hline
\end{tabular}

Website addresses are given for those databases that do not require a password. NHS employees can obtain a generic password for multiple bibliographic databases by registering with Athens via the National Electronic Library for Health (http://www.nelh.nhs.uk) or the NHS Scotland e-library (http://www.elib.scot.nhs.uk).

database relevant to family planning and reproductive health is Population Information Online (POPLINE). Table 1 lists those electronic bibliographic databases specific to health-related literature.

Electronic bibliographic databases hold vast amounts of literature, and a basic knowledge of Boolean operators (and/or/not) and other techniques can enhance your ability to retrieve relevant citations. Greenhalgh ${ }^{8}$ provides a useful overview of medical subject headings (MesH) and how to increase the sensitivity of a MEDLINE search. It is well worth undergoing a training session with an experienced medical librarian for tips on advanced retrieval features and professional advice on controlled versus free-text vocabulary. Consider other sources of information, e.g. grey literature (conference proceedings, government/ technical reports), reference checking, contact with authors of primary studies and hand-searching of key journals. When reading methodology sections of published systematic reviews think about whether authors have accessed all relevant information sources and which languages and time periods have been included.

\section{Assessment of methodological quality and summary of findings}

Once a comprehensive literature search has been conducted and articles have been retrieved and assessed for inclusion eligibility, the next stage is to critically appraise the articles to assess methodological quality. In addition, information should be extracted from each article in a standard manner to allow findings to be summarised and for inclusion in a meta-analysis if appropriate. Not all research has been conducted to the same rigour so it is important to include only valid research in the review. Are there major flaws in the research that comprise the bottom line finding? For example, a cohort study with large losses to follow-up will compromise the validity of findings. Similarly, a clinical trial comparing two sterilisation techniques that did not adopt random allocation or ensure adequate concealment of the allocation sequence is likely to be subject to selection bias. External validity relates to generalisability, or the 
extent to which results from a study can be generalised to other patients or populations. Consider a UK-wide representative survey undertaken to estimate chlamydia screening in genitourinary clinics; if a non-random sample of clinics are surveyed, results may be biased and not representative of the UK situation.

\section{Data extraction from included studies}

Details regarding the individual studies in the review should be available to journal readers. This will increase the objectivity of the review and allow readers to assess whether the review's conclusions are justified by the evidence available. A data abstraction form increases the objectivity of the review by ensuring that information abstracted is consistent from study to study. Key variables of interest may include study design, sample size, sample characteristics (age, sex, socio-economic status, smoking status), co-morbidity and outcomes of interest (prevalence rate, morbidity, mortality, quality of life, cost). Guidance to summarising the evidence is provided in Table 2 . The exact content of the evidence summary will depend on the question and study design adopted. A recent review of therapeutic acupuncture for gynaecological conditions included a table of data from individual studies. ${ }^{11}$ Results of systematic reviews are often presented by outcome(s) or study quality, with greater weight given to results from rigorous, well-conducted studies.

When multiple studies address the same question, the results can be numerically combined to provide an overall answer or pooled value. A recent systematic review by Kuyoh et al. ${ }^{12}$ compared the efficacy of diaphragms and vaginal sponges as contraceptives, with pregnancy rates and discontinuation rates as primary outcome measures. The authors presented results from two RCTs separately and calculated an overall result (odds ratio) for discontinuation rate at 12 months, concluding that sponge contraceptives are less effective than diaphragms at preventing pregnancy.

However, it may not always be appropriate to statistically pool results from individual studies, therefore meta-analysis should only be conducted when it is meaningful to do so. Many systematic reviews within public health, social sciences and education summarise results of primary studies using a qualitative (narrative) approach. ${ }^{13}$ It is always appropriate to systematically review a body of data, but may be misleading to numerically combine results. This is particularly so for data from systematic reviews of observational studies.

\section{Interpretation of findings and recommendations}

The overall interpretation of findings should be considered with respect to the strength of evidence, methodological quality of studies, consistency across studies, applicability to clinical practice and generalisability. Can policy decisions be made based on these findings? Is this the best available evidence we have to date? Although RCT studies are the most rigorous design, clinical and policy decisions are often made using the available evidence, which may be from observational studies, where no, few or methodologically flawed RCTs exist.

\section{Summary}

Although this Journal regularly accepts narrative rather than systematic reviews, the aim of this short paper is to encourage practitioners and researchers in family planning and reproductive health care to adopt a thorough and objective approach to literature reviews. It is hoped that readers appreciate what constitutes a systematic literature

Table 2 Example of evidence summary for included studies

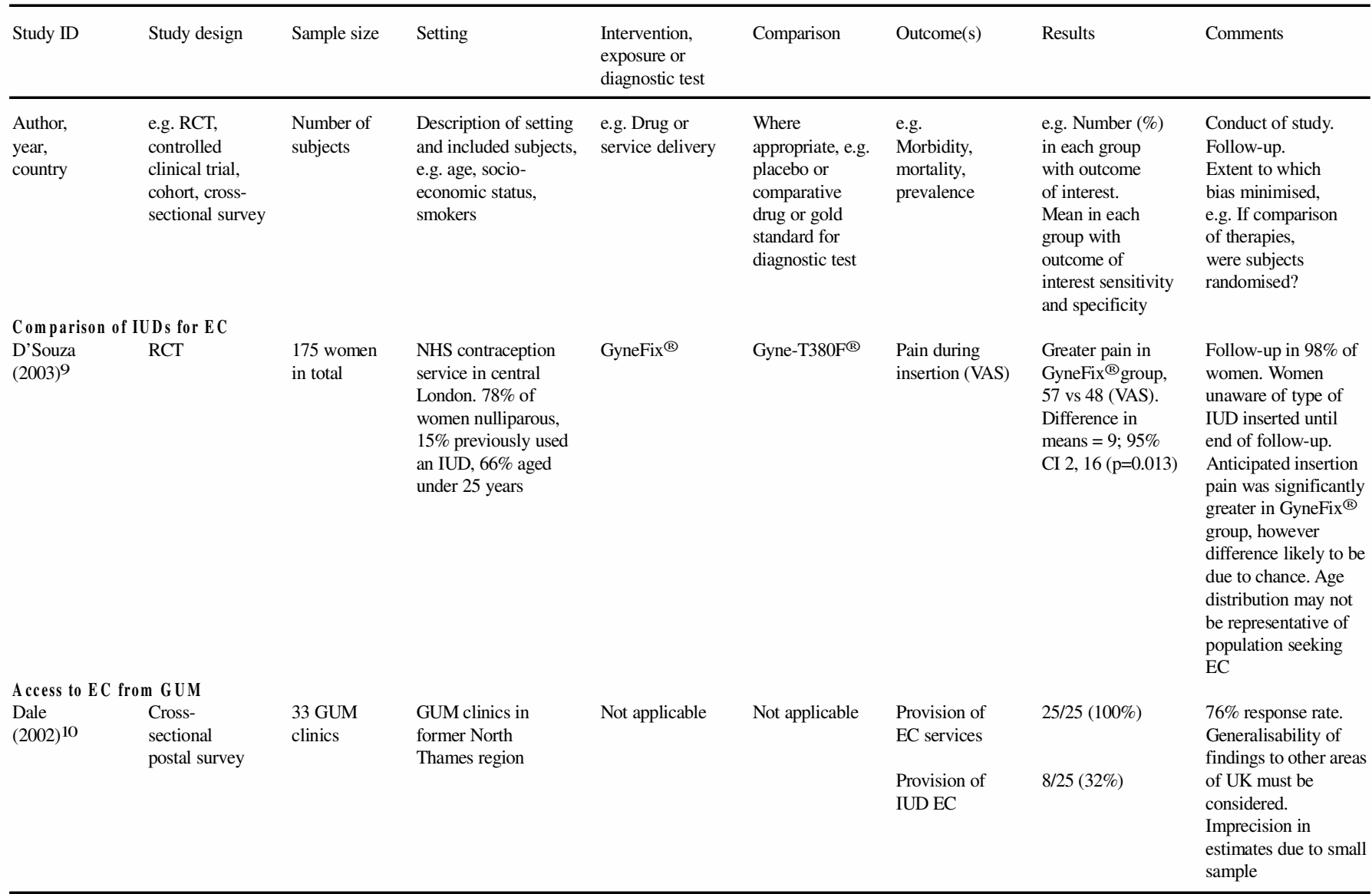

CI, confidence interval; EC, emergency contraception; GUM, genitourinary medicine; IUD, intrauterine device; RCT, randomised controlled trial; VAS, visual analogue scale, where 0 represents no pain at all and 100 the worst pain imaginable. 
review and their application in identifying current knowledge in family planning to facilitate practice. The systematic review overcomes much of the subjectivity of traditional narrative reviews by using an objective approach to reduce bias. The key stages in conducting a review outlined above should be considered, namely: comprehensive identification of relevant literature, explicit inclusion criteria, critical assessment of included studies, summarising data in an informative manner and interpretation of findings.

It is recommended that reviews be as systematic as is practical but there is often a trade-off to be considered between time and cost of conducting a review against resulting quality. Busy practitioners may not have the time or resources to conduct systematic reviews to the standards recommended by the Cochrane Collaboration ${ }^{14}$ or the NHS CRD. ${ }^{15}$ In practice, reviews span from subjective essays based upon personal opinion to 'Cochrane-style' systematic reviews. Nevertheless, we hope this paper emphasises the need for methodological rigour when literature reviewing and encourages practitioners to move along the continuum away from personal opinion towards objective, systematic reviews.

Statements on funding and competing interests

Funding. None identified.

Competing interests. None identified.

\section{References}

1 Deeks JJ. Systematic reviews of evaluations of diagnostic and screening tests. BMJ 2001; 323: 157-162.

2 Altman DG. Systematic reviews of evaluations of prognostic variables. BMJ 2001; 323: 224-228.
3 Cochrane AL. Effectiveness and Efficiency. Random Reflections on Health Services. London, UK: Nuffield Provincial Hospitals Trust, 1972.

4 Egger M, Davey Smith G, O'Rourke K. Rational, potentials and promise. In Egger M, Davey Smith G, Altman D (eds), Systematic Reviews in Health Care: Meta-analysis in Context (2nd edn). London, UK: BMJ Books, 2001.

5 Greenhalgh T. How to Read a Paper: The Basics of Evidence-based Medicine (2nd edn). London, UK: BMJ Books, 2001.

6 Crilly M, Foy R. Evidence-based family planning: finding answers to clinical questions. J Fam Plann Reprod Health Care 2003: 29(2): $48-52$.

7 Egger M, Ebrahim S, Davey Smith G. Where now for meta-analysis? Int J Epidemiol 2002; 31: 1-5.

8 Greenhalgh T. How to read a paper. The Medline database. BMJ 1997; 315: 180-183.

9 D'Souza RE, Masters T, Bounds W, et al. Randomised controlled trial assessing the acceptability of GyneFix ${ }^{\circledR}$ versus Gyne-T380S ${ }^{\circledR}$ for emergency contraception. J Fam Plann Reprod Health Care 2003; 29(2): 23-29.

10 Dale AWS, Wilkinson C, Forster GE, et al. Provision of Chlamydia trachomatis screening in family planning clinics and emergency contraception in genitourinary medicine clinics. A collaborative cross-specialty survey. J Fam Plann Reprod Health Care 2002; 28: $185-188$.

11 White AR. A review of controlled trials of acupuncture for women's reproductive health care. J Fam Plann Reprod Health Care 2003; 29(4): 233-236.

12 Kuyoh MA, Toroitich-Ruto C, Grimes DA, et al. Sponge versus diaphragm for contraception: a Cochrane Review. Contraception 2003; 67: $15-18$

13 Petticrew M. Systematic reviews from astronomy to zoology: myths and misconceptions. BMJ 2001; 322: 98-101.

14 Clarke M, Oxman AD (eds). Cochrane Reviewers' Handbook 4.2.0 (updated March 2003). http://www.cochrane.dk/cochrane/handbook/ handbook.htm [accessed 10 October 2003]

15 National Health Service Centre for Reviews and Dissemination. Undertaking Systematic Reviews of Research on Effectiveness. York, UK: York Publishing Services, University of York, 2001.
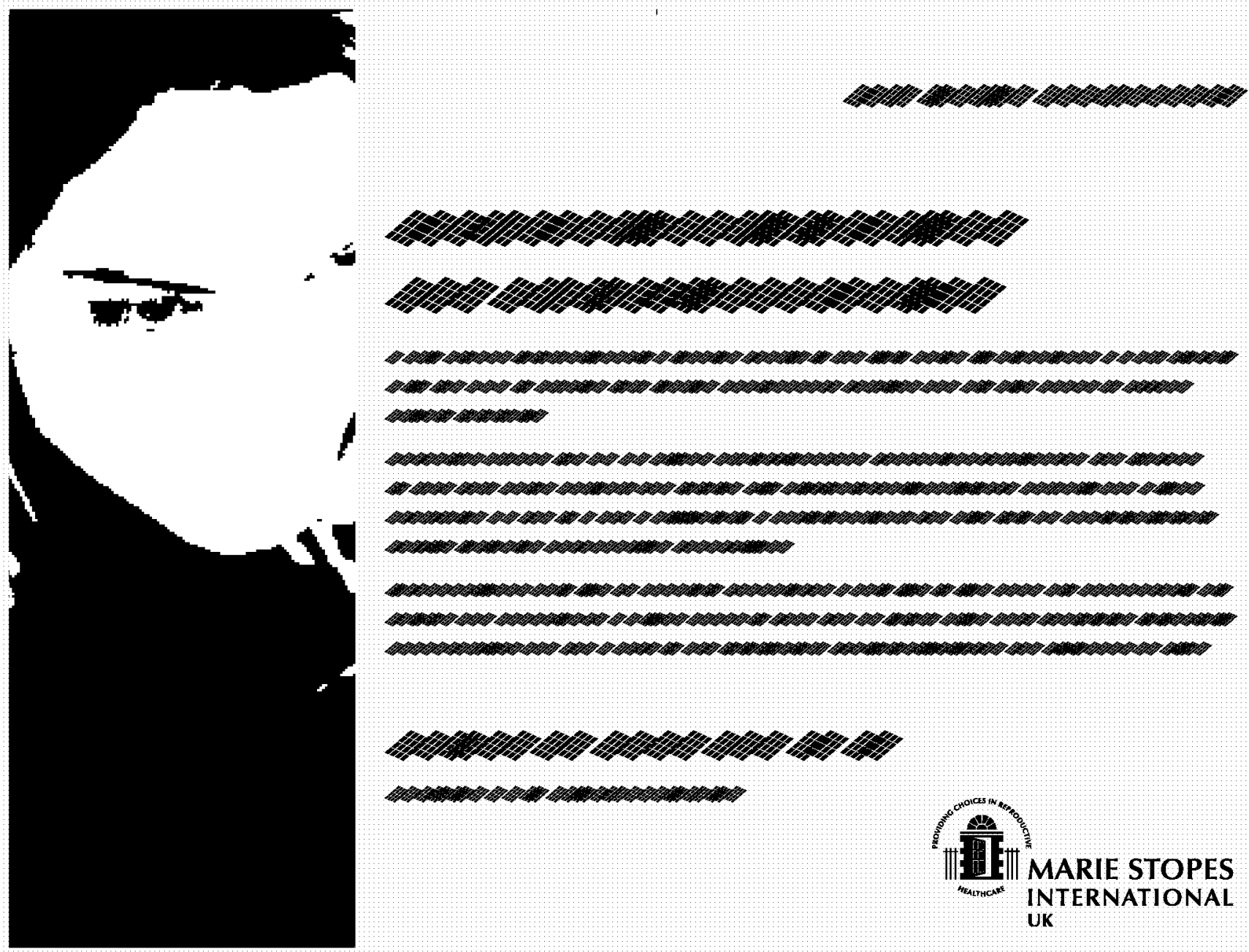
.

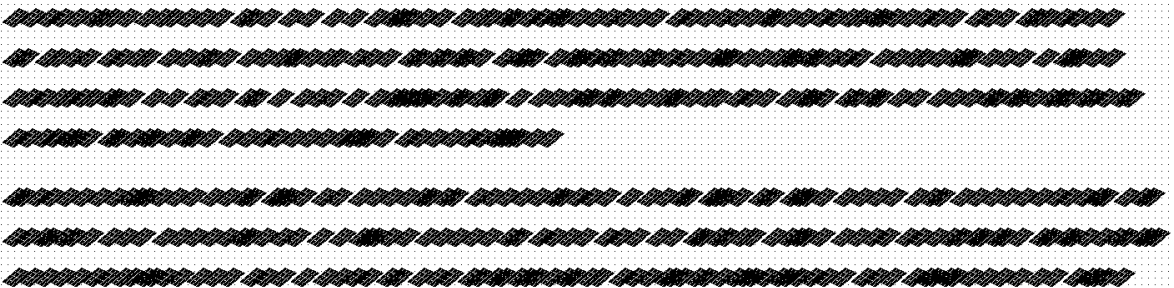

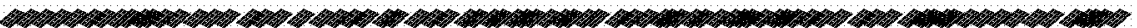
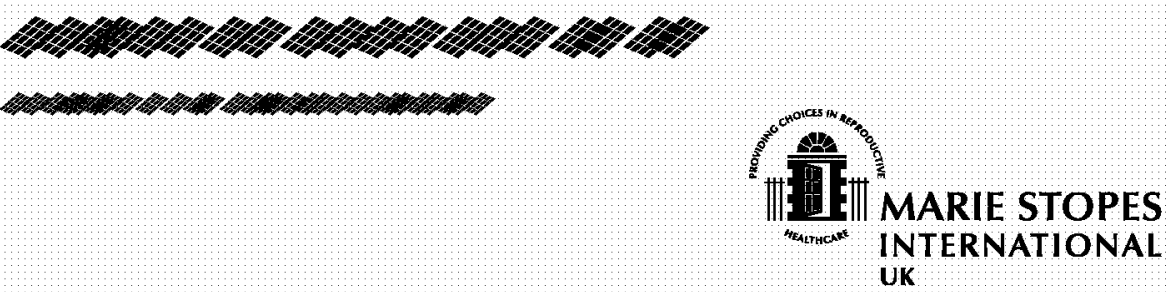